\title{
Probabilistic models to assist maintenance of multiple instruments
}

\author{
Joaquim Melendez, Beatriz Lopez \\ Institut d'Informàtica i Aplicacions \\ Universitat de Girona \\ Av. Lluis Santalo s/n \\ \{joaquim.melendez, beatriz.lopez\}@udg.edu
}

\author{
David Millán-Ruiz \\ Telefónica Investigación y Desarrollo \\ $\mathrm{C} /$ Emilio Vargas, 6 \\ dmr@tid.es
}

\begin{abstract}
The paper discusses maintenance challenges of organisations with a huge number of devices and proposes the use of probabilistic models to assist monitoring and maintenance planning. The proposal assumes connectivity of instruments to report relevant features for monitoring. Also, the existence of enough historical registers with diagnosed breakdowns is required to make probabilistic models reliable and useful for predictive maintenance strategies based on them. Regular Markov models based on estimated failure and repair rates are proposed to calculate the availability of the instruments and Dynamic Bayesian Networks are proposed to model cause-effect relationships to trigger predictive maintenance services based on the influence between observed features and previously documented diagnostics.
\end{abstract}

\section{Introduction}

This work has been motivated within the project AIMES (Advanced Infrastructures for Medical Equipment Supervision, UE-ITEA2). The project goals include the development of predictive mechanisms to assist maintenance of a large set of instruments submitted to an intensive use in healthcare institutions (hospitals). However, maintenance of a huge number of instruments is a challenge for large process industries (e.g. chemical, petrochemical or pharmaceutical) and also distribution industries spread across a wide area (e.g. electricity, gas, oil and water distribution) or networks of meteorological observatories with multiple (sometimes thousands) and heterogeneous devices submitted to an intensive use and rigorous maintenance and calibration scheduling. In all of these domains, specific maintenance policies are being applied to reduce cost and time associated to the downtimes of devices and the inclusion of reliable predictive mechanisms are strongly recommended.

Maintenance policies can be classified according to multiple criteria and strategies [1]. For example unit /multi-unit policies, reactive/periodic-preventive/ predictive policies or repair/replacement-non repair polices can be developed based on cost/benefit analysis. In this work, a scenario of multi-unit repairable devices is assumed and the aim is to develop a probabilistic approach to assist the implantation of predictive policies.

The intervention time with respect to equipment breakdown allows introducing three maintenance strategies: reactive, preventive and predictive maintenance. Reactive maintenance is associated with actions performed as a direct consequence of a breakdown. That is, once a failure has been produced the device is out of service and maintenance tasks consist in recovering its usage capabilities. On the other hand, preventive and predictive maintenance describe actions on the equipment previous to the occurrence of a breakdown. The aim of both is a reduction of costs and time associated to unexpected out of service equipment. The difference between them (preventive and predictive maintenance) is mainly associated to the cause that triggers these maintenance actions.

Preventive maintenance actions are associated to the existence of maintenance plan with scheduled actions to avoid possible breakdowns. Cleaning, substitution of consumables, calibration or equipment alternation, are some of these typical actions. Nevertheless, despite the existence of preventive maintenance policies breakdowns occur and predictive policies are being required to minimise the impact associated to the device unavailability. Predictive actions imply the existence of a monitoring system capable to sense and evaluate those devices on line in order to detect faults before occurring. In this paper, a probabilistic approach has been considered to create predictive models based on the exploitation of existing maintenance registers for the estimation of failure rates and internal cause-effect relationships to build those models. In the literature [2][3], several approaches to cope with this challenge have been described, that range from Markov models, Neural Networks, Regressive models, Decision Trees or Fuzzy models to Bayesian Networks (BN) or Influence Diagrams (ID). BNs provide a theoretical framework to include such information into a rigorous analysis and at same time exploiting their representation capabilities to give understandable models instead of the "black-box" solutions provided by neural networks or SVMs, which are usually not accepted in medical domains. 
The paper has been organised in five additional sections. Section 2 is devoted to define a representation for instruments. In section 3 and 4 probability models useful for instrument maintenance and their exploitation to assist predictive maintenance policies are introduced. Finally, conclusions are summarised in Section 5.

\section{Instrument representation}

A basic monitoring infrastructure is assumed to guarantee the connectivity of the instruments being monitored with the monitoring and maintenance applications in order to assure the existence of historical maintenance data. On line monitoring of internal variables and access to reports of autocheck units are examples of the observable data or symptoms, $o b s$. The evaluation of these observations as abnormal fires the necessity of a maintenance service who diagnoses the causes of the misbehaviour, or diagnostics, diag.

The purpose of this paper is to exploit information gathered in these diagnostic reports to model for each instrument the dependencies among the variables in the set $\{o b s, d i a g\}$. These models will be proposed as useful predictive mechanisms in presence of certain evidences or observed realisations of some variables in the set $\{o b s, d i a g\}$. Based on this issue, the following instantaneous representation, $I_{t}$, is proposed for a monitored instrument:

$I_{t}=\langle e, s, t\rangle$

where $e$ represents the set of observed evidences at the time instant $t$ and $s$ the corresponding status $(s=O K$, the instrument is under normal operating conditions; and $s=F$ indicates a failure requiring a maintenance intervention).

\section{Probability methods for instrument maintenance}

Markov modelling is a technique commonly used for reliability and safety analysis to represent models of non-repairable, partially repairable or fully repairable systems based on state diagrams [4]. Success and failure states are represented by circles and transitions between two states by directed arcs. Markov process is based on the assumption that the transition from one state to another one only depends on the current state and not on the previous states. In the context of maintenance, transitions from a successfully operating state to a maintenance state represent instantaneous failure rates $(\lambda)$ whereas the transitions from a maintenance service to a normal operating conditions represent a repair rate $(\mu)$. Thus, simple repairable devices submitted to a reactive maintenance policy can be represented by the simple graph Fig. 1a), where only two states are possible $(\mathrm{s}=\mathrm{OK} / \mathrm{F})$ and transitions between both are represented by failure rate, $\lambda_{O F}$, and the repair rate, $\mu_{O F}$. An enhanced model including mechanisms for triggering predictive actions is represented in Fig. 1b). In this diagram, D represents the instrument in the predictive maintenance state (transition rate $\lambda_{O D}$ ), from which the device would have to return to a normal operating condition (repair rate $\mu_{D O}$ ), but in certain occasion a breakdown, $\mu_{D F}$, could occur before the predictive intervention.
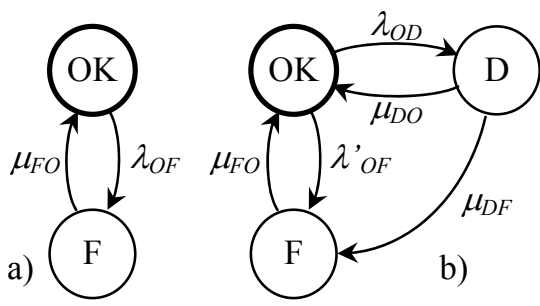

Fig. 1 State diagram: a) reactive maintenance b) predictive maintenance)

Predictive maintenance aims at cost reduction associated to unexpected breakdowns $\left(\lambda^{\prime}{ }_{O F}<\lambda_{O F}\right.$ in Fig. 1) by means of the inclusion of early detection mechanisms capable to trigger a predictive maintenance actions (possibly associated with degraded operating conditions).

\subsection{Reactive maintenance model creation}

Assuming that the device is submitted to a uniform constant strength and a random stress, failure rate in the previous model can be considered constant (exponential probability distribution function). Therefore, a feasible estimation can be obtained from a family of instruments submitted to the same use conditions by computing the following expression:

$\lambda_{\text {OF }}=\frac{\text { Number of Failures }}{\text { Total time exposed }}$

where Total time exposed is the addition of cumulative time associated to the instruments considered in the sample. For example, imagine that a set of 15 Vital-Signs monitors installed in the Intensive Care Unit (ICU) area of a hospital are used in this estimation and 5 of them failed during an observation time of 5 years. The failure rate estimation can be computed as $\lambda_{O F}=5 /(15 \times 5 \times 365)$ when a discrete time interval of 1 day is considered. Similarly a repair rate can be estimated if the average repair time is known (in the same time units) using the following expression:

$\mu_{F O}=\frac{1}{\text { Average repair time }}$

where the average repair time is also known as MTTR or Mean Time To Repair. 


\subsection{Predictive maintenance model creation}

The three-state model proposed in subsection 3.1 is a high-level representation of a possible predictive model whose main drawback resides in the estimation of $\lambda_{O D}$ before implementing predictive maintenance policies. Nevertheless, from the existing maintenance reports and repair worksheets is possible to establish relationships between evidences, $\hat{E}$, and the commonest failures. The exploitation of this information in a probabilistic graphical model, which represents a set of variables and their probabilistic independencies, can be easily done with BNs [5].

BNs can be applied in cases of uncertainty when certain probabilities are well-known and other unknown ones are tried to be obtained. Sometimes, a $\mathrm{BN}$ can be specified by a domain expert whilst, in other cases, this task is automatically done by a learning algorithm. In those cases, the network structure and its parameters are estimated from the database.

The result is a directed acyclic graph $G=(V, A)$ such that $A \subseteq[V]^{2}$ (the elements of $A$ are 2-element subsets of $V$ ). The elements of $V$ are the vertices of the graph $G$ and the elements of $A$ are its edges. In our case, the elements of $V$ represent concepts or events in the real world whereas the elements of $A$ symbolise the causal relationship between two vertices $v_{i}, v_{j} \in V$. The elements of $V$ can be divided into two groups: root nodes (without ancestors) which are expressed by a probability table and intermediate nodes, which are described by a conditional probability table.

The inclusion of a temporal representation in such a model leads to Dynamic Bayesian Networks (DBN). A $\mathrm{DBN}$ is basically a $\mathrm{BN}$ that represents sequences of variables. The simplest DBN would be a hidden Markov [8] model in which the state is not directly visible, while the output of a given state is observable. DBN are commonly proposed to predict and report potential risks which should be faced. Obviously, this can be also approached by using an ID but DBNs [6] usually behave faster in such an environment [7].

Within a DBN, the dynamic behaviours can be approached as a set of timeslices which describe the state of a given device at an instant, $t$. At this stage, we firstly need a representation for our DBN. Thus, let $X_{t}$ be a set of unobservable states at a time $t$ and $\hat{E}_{t}$, a set of observable evidences at a time $t$. In this way, the initial state can be expressed as $P\left(X_{0}\right)$. In order to simplify the problem, suppose that the transition rate $\lambda_{O D}$ follows a stationary process, in other words, its joint probability distribution does not change when shifted in time or space. Considering that values in any state are only influenced by the values of the state which directly precedes it (Markov assumption), previous states can be removed once a specified timeslice has been passed. Taking into account these considerations, transition model which describes temporal dependencies among states is defined as follows:

$P\left(X_{t} / X_{1: t-1}\right)=P\left(X_{t} / X_{t-1}\right)$

Note that, although unobservable states influence some variables, we do not need to directly know these states since we can define an observation model by reducing the ancestors of the evidence $\hat{E}_{t}$, as follows:

$$
P\left(\hat{\mathrm{E}}_{t} / X_{1: t}, \hat{\mathrm{E}}_{1: t-1}\right)=P\left(\hat{\mathrm{E}}_{t} / X_{t}\right)
$$

Idyllically, once the transition and observation models are defined for a given instant, a probability distribution which describes the current state of unobservable features can be inferred from current evidences and our knowledge from previous states. Unfortunately, an accurate solution for this inference problem rapidly becomes unfeasible. To mitigate this handicap, we propose the Boyen-Koller algorithm [8], BK, which requires low computing times and storage capabilities (this algorithm is briefly explained in subsection 4.2).

As example of the propose mechanism, a simplified vision of a Vital-Signs Monitor (VSM) with four potential failure causes or diagnostics (diag) is presented: Electricity $\{\mathrm{Ok}$, Fail $\}$, Oximeter $\{\mathrm{Ok}$, Fail $\}$, Blood Pressure Analyser $\{\mathrm{Ok}$, Fail $\}$ and Screen $\{\mathrm{Ok}$, Fail $\}$ which could cause VSM not to correctly work. This misbehaviour can be detected by observing 3 symptoms (obs): S1-VSM displays random numbers, S2- the monitor is in black and S3- the VSM is beeping; which depend on VSM's functioning. Then, the set of observable nodes, $V$, will be composed by 8 nodes. Also, consider two states, $X_{t}$ and $X_{t+1}$ (see Figure 2).

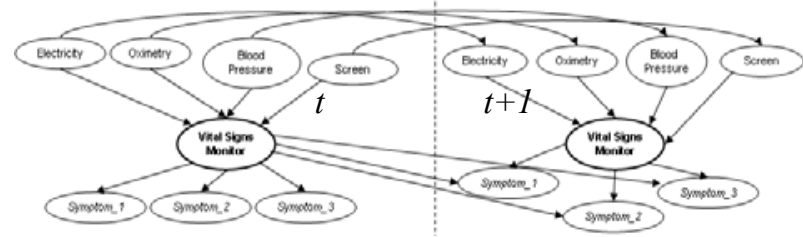

Fig. 2 DBN which models VSM's misbehaviours through two timeslices (from $t$ to $t+1$ ).

In our example, Electricity (El), Oximeter (O), Blood Pressure Analyser (B) and Screen (Sc) do not only modify the probability of having misbehaviours in the VSM, but also condition their future states. In this way, the transition model for the VSM is $P\left(V S M_{t} \mid V S M_{t-1}\right)$ and the observation model is $P\left(V S M_{t} / E l_{t}, O_{t}, B_{t}, S c_{t}\right)$. We can now consider binary states for the devices: $F$ means misbehaviour in the VSM, and $O K$ means that the VSM is completely operational [7]. If at time $t$, our evidence $\hat{E}_{t}$ is that Electricity $=$ Fail, then the $P\left(V S M_{t+1}=F\right)$ increases. The prediction from $t$ to $t+1$, applying the Markov property at $t+1$, is $P\left(V S M_{t+1} / E_{t}, E_{t+1}\right)$.

If our evidence $\hat{E}_{t}$ is that $\mathrm{S} 2=$ True, then the $\mathrm{P}\left(\mathrm{VSM}_{\mathrm{t}+\mathrm{i}}=\mathrm{F}\right)$ also increases but we would not know the causality since it might be caused by the nodes Electricity or Screen. For this reason, their probabilities 
$\mathrm{P}(\mathrm{El}=\mathrm{Fail})$ and $\mathrm{P}(\mathrm{Sc}=\mathrm{Fail})$ also increase. The prediction from $t$ to $t+1$ would be analogous to the previous example but respecting their conditional probabilities.

\section{Maintenance model exploitation}

\subsection{Availability analysis}

Since the states (ok / faulty) are directly visible this type of models are known as regular Markov models and the state transition probabilities are the only parameters. The previous models presented in 3 can be represented by the following transition matrix, P, (where the diagonal corresponds to the no change rate for each state) for Fig. 1a) and Fig. 1b) respectively:

$$
\begin{gathered}
P=\left[\begin{array}{cc}
1-\lambda_{O F} & \lambda_{O F} \\
\mu_{F O} & 1-\mu_{F O}
\end{array}\right] \\
P=\left[\begin{array}{ccc}
1-\lambda_{O F}^{\prime}-\lambda_{O D} & \lambda_{O F}^{\prime} & \lambda_{O D} \\
\mu_{F O} & 1-\mu_{O F} & 0 \\
\mu_{D O} & \lambda_{D F} & 1-\mu_{D O}-\lambda_{D F}
\end{array}\right]
\end{gathered}
$$

Transitions matrix, $P$, shows probabilities for moving from any one state to another state in one time interval or $n$ time intervals if $P^{n}$ is used instead. For $n \rightarrow \infty$ the limiting state is reached (the probability of going to a specific state is the same regardless of starting state). Limiting state can be used to compute the availability, $A V$, of the instrument; that is the probability that the device is successful at time $t$. It is computed by adding the success limiting states probability [4]. Assuming that the initial state is $s=O K$ the following expression can be used to compute availability of Fig. 1:

$$
A V=\left[\begin{array}{lll}
1 & 0
\end{array}\right] P^{n}
$$

where $n$ is the day when the availability is going to be evaluated. For $n \rightarrow \infty$ the steady state availability is obtained.

\subsection{Failure prediction}

Boyen-Koller algorithm [8], BK algorithm represents the belief state as a product of marginals over clusters. The main steps of this algorithm are the following ones:

1. Choose a tractable representation for an approximate belief state. BK scheme assumes independence among factors. In our example, every feature could be a valid factor of BK (4 clusters). If we had another feature pointing to Electricity (an ancestor), then it would belong to its cluster too.

2. Propagate the belief state through the transition model at the instant $t$ and condition it, according to $t+1$.
3. Continue approximating the belief state.

The application of this algorithm is suggested in the exploitation step.

\section{Conclusions and Future Work}

The paper recovers existing approaches to integrate in a common framework different maintenance policies based on the exploitation and reuse of useful information generated through daily maintenance activity. Probability models have been proposed to assist maintenance policies and simple regular models have been introduced to model reactive and predictive maintenance. The impossibility to estimate transition rates from normal operating conditions to predictive states has been overcome with the use of DBN. BN allows for representing independency among variables, including those that are observables but also the variables describing fault causes; and these extensions, DBN, allows modelling the temporal dimension.

For the evaluation of such a methodology, a selection of instruments submitted to an intensive use, e.g. VSM, will be used. Historical maintenance registers are proposed to estimate the involved parameters and DBN topology and probabilities. Cross validation will be used to evaluate performance with the available data and confusion matrix based indices (e.g. sensitivity, precision or accuracy) will be used to rank the method.

\section{Acknowledgement}

This proposal is being developed in the framework of the AIMES EU-ITEA2 Project, encouraged by the German BMBF (Ref. 01ISO8001E) and Spanish Avanza I+D program (ref. TSI-020400-2008-47).

\section{References}

[1] H. Wang. "A survey of maintenance policies of deteriorating systems". European Journal of Operational Research, Vol. 139, pp. 469-489, 2002.

[2] Pelaez, J.R. et al. "Predictive maintenance oriented neural network system - PREMON“. I.E.S., p. 49 - 52, 2001.

[3] L. Yan and L. Shi-qi. "Decision Support for Maintenance Management Using Bayesian Networks". WiCom, 2007.

[4] W.M. Goble, "Control Systems. Safety Evaluation and Reliability", 2n edition, ISA, 1998.

[5] E. Castillo, J.M. Gutiérrez, and A.S. Hadi. "Expert Systems and Probabilistic Network Models". New York, 1997.

[6] K. Murphy. "Dynamic Bayesian Networks: Representation, Inference and Learning". PhD thesis, U.C. Berkeley, 2002.

[7] D. Ozgur-Unluakin and T. Bilgiç. Predictive "Maintenance using Dynamic Probabilistic Networks". Probabilistic Graphical Models, p. 239-246, 2006.

[8] L.R. Rabiner. "A tutorial on Hidden Markov Models and selected applications in speech recognition". Proceedings of the IEEE, pp. 257-286, 1989.

[9] X.R Boyen and D. Koller. "Tractable Inference for Complex Stochastic Processes." XIV Conference on Uncertainty in Artificial Intelligence (UAI), pp. 33-42, 1998. 